\title{
Séminaires / Seminari 2012
}

\author{
Ouverture et reprise d'un cabinet \\ médical
}

\section{Contenu}

Business plan (préparation du plan de financement et crédit d'exploitation, financement par la banque), Aménagement (implantation, projet et concept d'aménagement, choix du mobilier, budget), Estimation d'un cabinet (inventaire et goodwill), Administration d'un cabinet médical (dans le cabinet, par la banque), Assurances (toutes les assurances à l'intérieur et autour du cabinet), Passage du statut de salarié à celui d'indépendant et fiscalité.

\section{Sponsors}

Les coûts sont pris en charge par divers sponsors (voir www.fmhservices.ch).

\section{Dates}

\begin{tabular}{|lll}
\hline K20 & $\begin{array}{l}\text { Jeudi } 8 \text { mars } 2012 \\
13.30-18.00 ~ h\end{array}$ & $\begin{array}{l}\text { Lausanne } \\
\text { World Trade Center }\end{array}$ \\
\hline K21 & $\begin{array}{l}\text { Jeudi } 31 \text { mai } 2012 \\
13.30-18.00 ~ h\end{array}$ & $\begin{array}{l}\text { Genève } \\
\text { Crowne Plaza }\end{array}$ \\
\hline
\end{tabular}

\section{Remise d'un cabinet médical}

\section{Contenu}

Aspects juridiques (autour du contrat de remise/reprise), Estimation d'inventaire et goodwill d'un cabinet, Assurances (prévoyance, assurances à l'intérieur et autour du cabinet), Conséquences fiscales d'une remise.

\section{Sponsors}

Les coûts sont pris en charge par divers sponsors (voir www.fmhservices.ch).

\section{Dates}

\begin{tabular}{|c|c|c|}
\hline K24 & $\begin{array}{l}\text { Jeudi } 10 \text { mai } 2012 \\
17.00-21.30 \mathrm{~h}\end{array}$ & $\begin{array}{l}\text { Lausanne } \\
\text { World Trade Center }\end{array}$ \\
\hline K25 & $\begin{array}{l}\text { Jeudi } \\
15 \text { novembre } 2012 \\
17.00-21.30 \mathrm{~h}\end{array}$ & $\begin{array}{l}\text { Genève } \\
\text { Crowne Plaza }\end{array}$ \\
\hline
\end{tabular}

\section{Cabinet de groupe}

\section{Contenu}

Esprit d'entreprise: Traite des différences entre un cabinet de groupe et un cabinet individuel, et des différentes gestions (entreprise, collaborateurs). Quelles sont les exigences envers le personnel et comment s'organise la planification d'un cabinet de groupe en fonction d'un projet?

Modèles de cabinet / formes juridiques / finances / fiscalité: Cette partie sera consacrée à la présentation transparente des différents modèles de cabinets et des formes juridiques possibles (cabinet individuel, système de partenariat junior-senior, infrastructure SA / S.A.R.L., solution SA / S.A.R.L. avec intégration globale) et à l'explication des différentes formes de financement du point de vue financier et de la fiscalité. Elle traitera également la question de la gestion et de l'utilisation des moyens financiers et abordera la planification fiscale stratégique et opérationnelle (y. c. la question de la TVA dans les cabinets de groupe).

Assurances / Prévoyance professionnelle: Quelles sont les principales différences en matière de prévoyance entre une personnalité juridique (SA, S.A.R.L) et une entreprise individuelle? Quels sont les différents besoins individuels (par ex. médecin avec ou sans enfants) en matière de couverture d'assurance et de prévoyance professionnelle dans un cabinet de groupe? Quels aspects d'assurance responsabilité civile faut-il prendre en compte (qui doit conclure quoi et quand?) et quelles sont les implications fiscales pour une personne juridique? Qu'en est-il de la prévoyance privée?

Rapport d'expérience: Pour clore le séminaire, un médecin (co-fondateur d'un cabinet de groupe) de la région fait part de ses expériences quant à la création, les difficultés (où, quand et comment un soutien externe est nécessaire), l'organisation et les processus de gestion d'un cabinet de groupe.

\section{Sponsors}

Les coûts sont pris en charge par divers sponsors (voir www.fmhservices.ch).

\section{Date K85

$\begin{array}{ll}\text { Jeudi } & \text { Lausanne } \\ \text { 20 septembre 2012 } & \begin{array}{l}\text { World Trade } \\ \text { 13.30-18.00 h }\end{array} \\ \text { Center }\end{array}$

Apertura e rilevamento di uno studio medico

\section{Contenuto}

Business plan (preparazione del piano di finanziamento e del credito d'esercizio, prestito bancario), Pianificazione (insediamento, progetto e pianificazione, scelta del mobilio, budget), Valutazione di uno studio medico (inventario e goodwill), Amministrazione di uno studio medico (interna allo studio, rapporti con la banca), Assicurazioni (tutte le assicurazioni necessarie interne ed esterne allo studio), Passaggio dallo stato di dipendente a quello di indipendente, fiscalità.

\section{Documentazione}

Basato su una check-list per l'apertura di uno studio medico, il raccoglitore, consegnato ad ogni partecipante, contiene tutte le informazioni utili durante e dopo il seminario.

\section{Sponsor}

Diversi sponsor si fanno carico delle spese (si rimanda al sito www.fmhservices.ch).

\section{Date}

\begin{tabular}{|lll|} 
K50 & $\begin{array}{l}\text { Martedì } 6 \text { marzo 2012 } \\
\text { dalle } 17 \text { alle 21.00 }\end{array}$ & $\begin{array}{l}\text { Chiasso } \\
\text { FMH Fiduciaria } \\
\text { Services }\end{array}$ \\
\hline K51 & $\begin{array}{l}\text { Mercoledì } \\
16 \text { maggio } 2012 \\
\text { dalle } 14.00 \text { alle } 18.00\end{array}$ & $\begin{array}{l}\text { Chiasso } \\
\text { FMH Fiduciaria } \\
\text { Services }\end{array}$ \\
\hline
\end{tabular}

\section{Inscription et information /}

\section{Iscrizioni e informazioni}

www.fmhservices.ch ou FMH Consulting Services, Cornelia Steinmann, Burghöhe 1, 6208 Oberkirch, Tél. 04192500 77, Fax 041 9210586.

\section{Remarque / Osservazioni}

Les adresses des participants aux séminaires dont les coûts sont couverts en partie ou totalement par des sponsors sont communiquées aux sponsors concernés.

Gli indirizzi dei partecipanti ai seminari, i cui costi sono coperti in parte o completamente da degli sponsor, vengono comunicati agli sponsor interessati.

\section{Conditions d'annulation /}

\section{Condizioni d'annullamento}

Un montant est perçu pour une absence ou une annulation. Il est de:

Un importo verrà rimborsato in caso di assenza o annullamento. Esso sarà di:

- 50 CHF par personne dans les 15 jours avant le début du séminaire/ per persona entro i 15 giorni prima dell'inizio del seminario;

- 100 CHF par personne dans les 7 jours avant le début du séminaire / per persona entro i 7 giorni prima dell'inizio del seminario. 